\title{
Telomere association of Oryza sativa telomere repeat-binding factor like 1 and its roles in telomere maintenance and development in rice, Oryza sativa L.
}

\author{
Mi Young Byun ${ }^{1}$, Li Hua Cui ${ }^{2}$, Hyoungseok Lee ${ }^{1} \mathcal{E}$ Woo Taek Kim ${ }^{2}$ * \\ ${ }^{1}$ Unit of Polar Genomics, Korea Polar Research Institute, Incheon 21990, ${ }^{2}$ Department of Systems Biology, College of Life Science and \\ Biotechnology, Yonsei University, Seoul 03722, Korea
}

\begin{abstract}
Telomeres are specialized nucleoprotein complexes that function to protect eukaryotic chromosomes from recombination and erosion. Several telomere binding proteins (TBPs) have been characterized in higher plants, but their detailed in vivo functions at the plant level are largely unknown. In this study, we identified and characterized OsTRFL1 (Oryza sativa Telomere Repeat-binding Factor Like 1) in rice, a monocot model crop. Although OsTRFL1 did not directly bind to telomere repeats (TTTAGGG) $)_{4}$ in vitro, it was associated with telomeric sequences in planta. OsTRFL1 interacted with rice TBPs, such as OsTRBF1 and RTBP1, in yeast and plant cells as well as in vitro. Thus, it seems likely that the association of OsTRFL1 with other TBPs enables OsTRFL1 to bind to telomeres indirectly. T-DNA inserted OsTRFL1 knock-out mutant rice plants displayed significantly longer telomeres $(6-25 \mathrm{~kb})$ than those $(5-12 \mathrm{~kb})$ in wild-type plants, indicating that OsTRFL1 is a negative factor for telomere lengthening. The reduced levels of OsTRFL1 caused serious developmental defects in both vegetative and reproductive organs of rice plants. These results suggest that OsTRFL1 is an essential factor for the proper maintenance of telomeres and normal development of rice. [BMB Reports 2018; 51(11): 578-583]
\end{abstract}

\section{INTRODUCTION}

Telomeres, which are present at the ends of linear eukaryotic chromosomes, are essential for the preservation of genome integrity (1). Telomeres are tandem arrays of G/C-rich repeats and are associated with several telomere binding proteins

${ }^{*}$ Corresponding author. Tel: +82-2-2123-2661; Fax: +82-2-3125657; E-mail: wtkim@yonsei.ac.kr

https://doi.org/10.5483/BMBRep.2018.51.11.122

Received 2 June 2018, Revised 22 June 2018, Accepted 25 June 2018

Keywords: Developmental growth, Double-stranded telomere binding proteins, Protein-protein interaction, Rice (Oryza sativa L.), Telomere lengthening
(TBPs), which play critical roles in the protection and stabilization of chromosomal ends $(2,3)$. There are two distinct types of TBPs. One type is a group of single-stranded DNA-binding proteins that bind to the $3^{\prime}$ overhang sequences at telomere termini and are required for chromosome capping and telomerase regulation (4). The other group of TBPs, which includes human TRF1/PIN2 and TRF2 and yeast Rap1 and Taz1, bind to duplex telomeric DNA and regulate telomere length and structure (5). In human, shelterin is a telomereprotein complex composed of six proteins, POT1, TRF1, TRF2, TIN2, TPP1, and Rap1 (6). This complex forms a defined structure that prevents telomeres from being recognized as damaged DNA $(7,8)$.

In plants, double-stranded telomere binding proteins (DS-TBPs) are classified according to their structural characteristics $(3,9)$. Three distinct classes of DS-TBPs have been identified and characterized in higher plants, all of which contain a single telobox Myb domain (10). The first class harbors a Myb domain at the $\mathrm{N}$-terminal end and a linker histone $\mathrm{H} 1 / \mathrm{H} 5$ motif in the central region (11). Arabidopsis AtTRB1, AtTRB2, and AtTRB3, rice OsTRBFs, and maize $\mathrm{ZmSMH}$ belong to this group of proteins, which specifically binds to plant double-stranded telomeric repeats (TTTAGGG) (12-16). The second group is typified by a single Myb-like DNA-binding domain and an additional Myb-extension in the C-terminal region. Structural analysis of the telomere-binding properties of tobacco NgTRF1 and rice RTBP1 suggested that this plant-specific C-terminal Myb extension is required for the interaction of NgTRF1 and RTBP1 with telomeric sequences $(17,18)$. The expression level of NgTRF1 is closely linked to cell viability and the maintenance of telomere length and stability of tobacco BY-2 suspension cultured cells (19). Loss-of-function rtbp1 knockout mutant allele exhibited developmental defects, accompanied by genome instability in rice (20). These results suggested that NgTRF1 and RTBP1 DS-TBPs are functional components that have crucial roles in the maintenance of genome stability in tobacco and rice plants.

The third group of DS-TBPs contains a Myb DNA-binding domain in the C-terminus, without the C-terminal extension, 
resulting in different binding capacities to telomeric repeats in vitro (21). Arabidopsis contains six telomere repeat binding factor-like (TRFL) proteins, which belong to this third group. A gel retardation assay showed that the Arabidopsis TRFLs were unable to bind to telomeric repeats in vitro; thus, their biochemical and physiological roles remain to be elucidated (21).

In this study, we identified and characterized OsTRFL1, a rice TRFL protein. OsTRFL1 failed to directly interact with plant telomeric repeats (TTTAGGG) $)_{4}$ in vitro. However, a chromatin immuno-precipitation (ChIP) assay showed that OsTRFL1 is associated with telomeric repeats in planta. Yeast-two hybrid, in vitro pull-down, and bimolecular fluorescence complementation (BiFC) assays showed that OsTRFL1 interacted with the rice DS-TBPs OsTRBF1 and RTBP1. T-DNA inserted ostrfl1 knockout mutant rice plants displayed severe developmental abnormalities and failed to produce functional seeds. These results suggest that OsTRFL1 functions in telomere maintenance by interacting with other DS-TBPs in rice.

\section{RESULTS AND DISCUSSION}

\section{Identification and characterization of OsTRFL1}

Although Arabidopsis TRFL proteins were previously identified, their in vivo roles are unknown (21). A database search revealed a putative TRFL gene in rice, which we named Oryza sativa Telomere Repeat-binding Factor Like 1 (OsTRFL1; XP 015625825.1). Total RNA was prepared from rice leaves, and full-length OsTRFL1 CDNA was obtained by RT-PCR. The coding region of OsTRFL1 is $1869 \mathrm{bp}$, encoding 623 amino acids (molecular mass, 69 kDa; Fig. 1A). OsTRFL1 possesses a single DNA-binding Myb domain at its C-terminal end. The Myb-extension was absent in OsTRFL1. Amino acid sequence alignment showed that the Myb domain of OsTRFL1 is highly conserved with those of Arabidopsis and rice DS-TBPs (Supplementary Fig. S1). As OsTRFL1 contains the conserved DNA-binding Myb motif, the binding affinity of OsTRFL1 to telomeric repeats was examined. A bacterially-expressed Myc-(His) ${ }_{6}$-OsTRFL $1^{486-623}$ recombinant protein, which contains the Myb DNA-binding motif (Supplementary Fig. S2), was incubated with plant telomere repeats (TTTAGGG) ${ }_{4}$, and its binding affinity was examined by a gel-retardation assay. As shown in Fig. 1B, Myc-(His) 6 -OSTRFL1 ${ }^{486-623}$ failed to interact with the telomere repeat sequence in vitro. In contrast, (His) ${ }_{6}-\mathrm{OsTRBF} 1^{1-128}$ and Myc-(His) ${ }_{6}-\mathrm{RTBP}^{506-620}$ showed high binding affinities for the telomeric repeats, as reported previously $(14,18)$.

Next, a ChIP assay was conducted to investigate the possible in vivo association of OSTRFL1 with telomeric DNA. OsTRFL1-sGFP, OsTRBF1-sGFP, OsRAD51D-sGFP, and RTBP1-sGFP fusion proteins were expressed in tobacco leaf epidermal cells using an Agrobacterium-mediated infiltration method. The nuclear genomic DNA-protein complex was extracted and subjected to immunoprecipitation with an anti-GFP antibody. The immunoprecipitated DNA was then hybridized with a ${ }^{32}$ P-labeled (TTTAGGG) 70 telomere repeat probe. The results showed that OsTRFL1 is associated with telomeric sequences in planta (Fig. 1C). However, the signal for OsTRFL1 was not as strong as the signals for OsTRBF1 and RTBP1, indicating that OsTRFL1 binds to telomeres indirectly. OsRAD51D was used as a negative control in the ChIP assay. As previously reported (22), OsRAD51D showed no binding to telomere repeats. Thus, it seems likely that OsTRFL1 is not able to directly bind to telomeric repeats, but it interacts with telomeres indirectly in plant cells.

\section{OsTRFL1 interacts with rice the DS-TBPs OsTRBF1 and RTBP1 in yeast cells and in vitro}

Numerous TBPs associate with telomeres to maintain the proper function and structure of chromosomal ends (23-25). Although OsTRFL1 failed to bind directly to the telomeric sequences in vitro, it was associated with telomere repeats in vivo (Fig. 1). These results raised the possibility that OsTRFL1

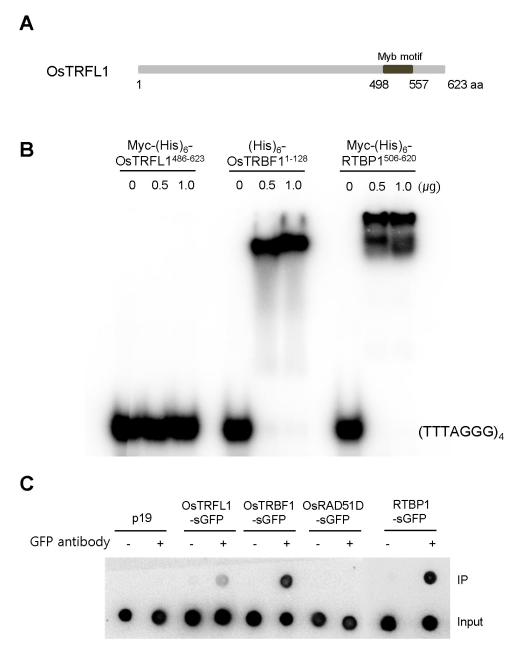

Fig. 1. OsTRFL1 is associated with double-stranded telomeric repeats in planta. (A) Schematic representation of rice OsTRFL1. The C-terminal Myb DNA-binding motif is indicated. (B) Gel retardation assay. Different amounts $(0,0.5$, and $1.0 \mu \mathrm{g})$ of bacterially-

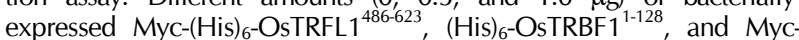
(His) ${ }_{6}-\mathrm{RTBP}^{506-620}$ fusion proteins, all of which possess the Myb DNA-binding domain, were incubated with ${ }^{32}$ P-labeled (TTTAGGG) telomere repeats. After incubating for $10 \mathrm{~min}$ on ice, the reaction mixtures were loaded on an $8 \%$ non-denaturing polyacrylamide gel. (C) ChIP assay of OsTRFL1. The 35S:OsTRFL1-sGFP, 35S:OsTRBF1-sGFP, 35S:RTBP1-sGFP, and 35S:OsRAD51D-sGFP constructs were introduced into tobacco leaves by Agrobacteriummediated infiltration. After 2 days of infiltration, nuclear genomic DNA-protein complexes were isolated and subjected to immunoprecipitation with an anti-GFP antibody. The pull-downed DNA was hybridized with a ${ }^{32}$ P-labeled (TTTAGGG) $)_{70}$ telomere repeat probe. IP, immunoprecipitation. 
interacts with telomeres indirectly in the presence of other TBPs. Therefore, we tested the interaction of OsTRFL1 with two rice DS-TBPs, OsTRBF1 and RTBP1 (Fig. 2A). A yeast-two hybrid assay showed that OsTRFL1 interacts with both OsTRBF1 and RTBP1 (Fig. 2B). OsTRFL1 also formed a homo-dimeric complex in yeast cells. In contrast, OsTRFL1 did not interact with OsKu70, which does not bind to telomeres (26). To investigate the direct interactions of these DS-TBPs, we conducted an in vitro pull-down assay. As shown in Fig. 2C, OsTRFL1 formed hetero-dimeric complexes with OsTRBF1 and RTBP1 as well as homo-dimer in vitro.

\section{In vivo interactions of OsTRFL1 with OsTRBF1 and RTBP1}

Because RTBP1 and OsTRBF1 have high affinities for telomeric sequences (Fig. 1B), these DS-TBPs are expected to

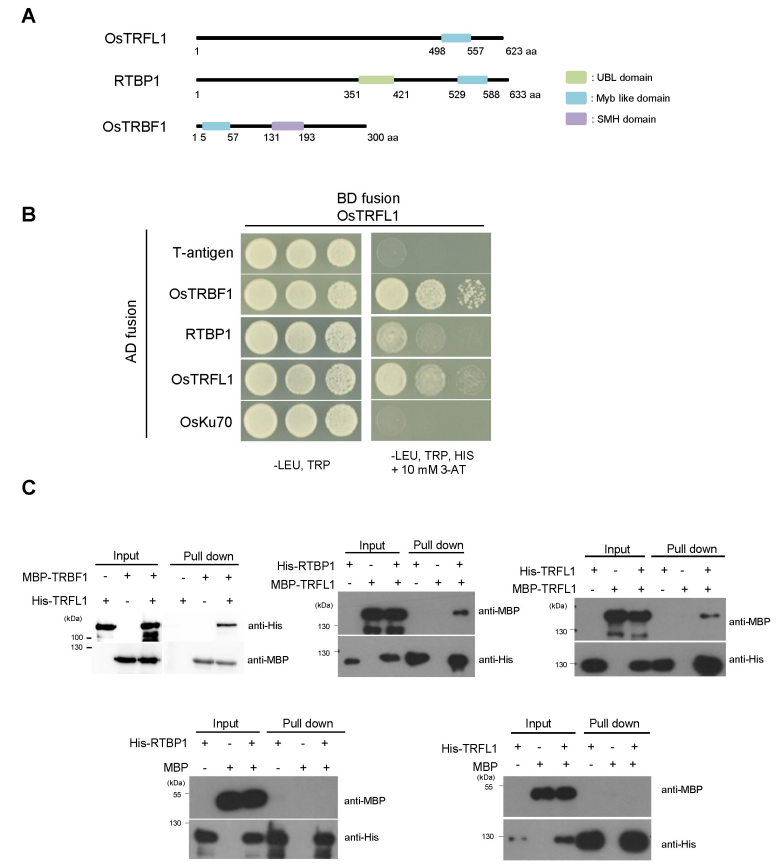

Fig. 2. OsTRFL1 interacts with rice DS-TBPs in yeast cells and in vitro. (A) Structural comparison of OsTRFL1, RTBP1, and OsTRBF1. The UBL domain, Myb DNA-binding motif, and SMH domain are indicated. (B) Yeast-two hybrid assay. OsTRFL1 was cloned into the pGBK T7 vector, and OsTRBF1, RTBP1, OsTRFL1, OsKu70, and T-antigen were cloned separately into the pGAD T7 vector. Each combination of the indicated plasmids was co-transformed into yeast $\mathrm{AH} 109$ cells. To test for protein-protein interactions, yeast cells were plated onto two-minus (-Leu/-Trp; left panel) or three-minus $(-\mathrm{Leu} /-\mathrm{Trp} /-\mathrm{His})$ medium containing $10 \mathrm{mM}$ 3-AT (right panel) and grown at $30^{\circ} \mathrm{C}$ for 4 days. T-antigen was used as a negative control. (C) In vitro pull-down assay. OsTRFL1, OsTRBF1, and RTBP1 DS-TBPs were expressed as MBPor $(\mathrm{His})_{6}$-fusion proteins in $E$. coli. The purified fusion proteins were co-incubated as indicated in the presence of His-affinity matrix. The bound protein was then eluted from the resin and immunoblotted with either anti-MBP or anti-His antibody. be localized to the nucleus. A subcellular localization assay using tobacco infiltration system revealed that both RTBP1-sGFP and OsTRBF1-sGFP do not display uniform distributions, but instead show speckle-like localization patterns in the nucleus (Fig. 3A). OsTRFL1-sGFP also showed a similar localization pattern that is speckle-like structures inside the nuclei. In addition, mRFP-OsTRFL1 was co-localized with RTBP1-sGFP and OsTRBF1-sGFP to the nuclear speckle-like bodies (Fig. 3B).

The in planta interactions of OsTRFL1 with RTBP1 and OsTRBF1 were tested by BiFC assay. For this assay, OsTRFL1 was fused to the $155 \mathrm{~N}$-terminal amino acids of yellow

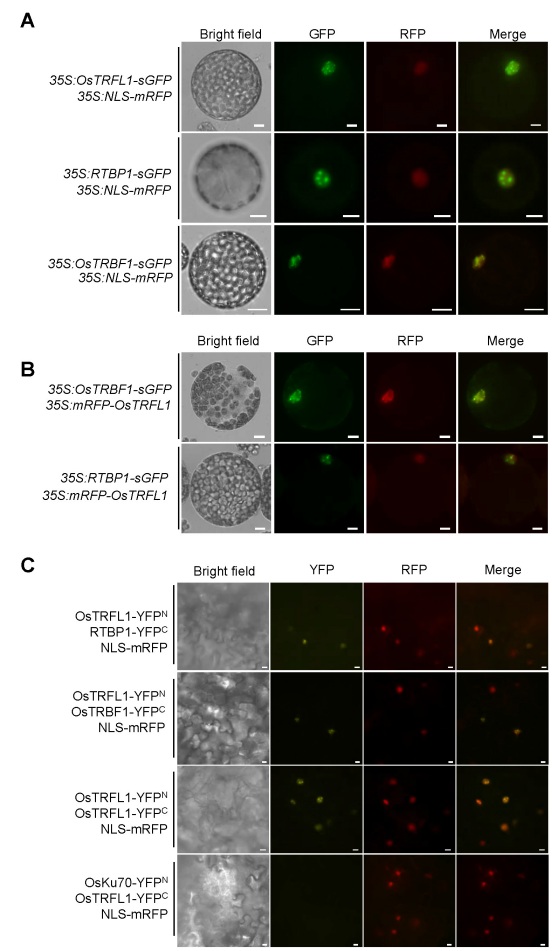

Fig. 3. OsTRFL1 interacts with rice DB-TBPs in nuclear specklelike structures. (A) The 35S:OsTRFL1-sGFP, 35S:RTBP1-sGFP, and 35S:OsTRBF1-sGFP constructs were co-expressed with 35:NLS$m R F P$ in tobacco leaves by Agrobacterium-mediated infiltration. After two days of infection, protoplasts were prepared from the infected leaves, and fluorescent protein signals were visualized by fluorescence microscopy. NLS-mRFP was used as a nuclear marker protein. Scale bars $=5 \mu \mathrm{m}$. (B) 35S:OsTRBF1-sGFP or 35S:RTBP1-sGFP was co-infiltrated with 35S:mRFP-OsTRFL1 into tobacco leaves. Fluorescent signals in the protoplasts were visualized by fluorescence microscopy. Scale bars $=5 \mu \mathrm{m}_{\text {. }}$ (C) BiFC assay. OsTRFL1-YFP ${ }^{N}+$ RTBP1-YFP $^{\text {, OSTRFL1-YFP }}{ }_{+}^{N}$ OsTRBF1-YFP ${ }^{C}$ and OsTRFL1-YFP ${ }^{N}+$ OsTRFL1-YFP $^{C}$ proteins were transiently expressed in three-week-old tobacco leaf cells. After 3 days of infiltration, the fluorescent signals were detected by fluorescence microscopy. OsKu70-YFP ${ }^{N}+$ OsTRFL1-YFP $^{C}$ was included as a negative control. NLS-mRFP was used as a nuclei-localized marker protein. Scale bars $=5 \mu \mathrm{m}$. 
fluorescent protein $\left(\mathrm{YFP}^{\mathrm{N}}\right)$, and OsTRBF1 and RTBP1 were fused to the $86 \mathrm{C}$-terminal amino acids of YFP (YFP ${ }^{\mathrm{C}}$ ). The fusion constructs were co-expressed in tobacco leaf cells by Agrobacterium-mediated infiltration. Mesophyll cells of the infiltrated leaves were visualized by fluorescence microscopy. Strong YFP fluorescent signals were detected in nuclear speckle-like structures when OsTRFL1-YFP ${ }^{N}+$ RTBP1-YFP $^{C}$ and OSTRFL1-YFP ${ }^{N}+$ OsTRBF1-YFP ${ }^{\mathrm{C}}$ fusion constructs were co-expressed, suggesting in planta interaction of OsTRFL1 with RTBP1 and OsTRBF1 (Fig. 3C). In addition, homo-dimeric formation of OsTRFL1 was detected as a speckle-like pattern within the nuclei. Again, there was no detectable signal when OsKu70-YFP ${ }^{N}$ and OsTRFL1-YFP ${ }^{\mathrm{C}}$ were co-expressed, suggesting that OsKu70 and OsTRFL1 do not interact with each other in vivo. Taken together, the results presented in Figs. 1-3 suggest that OsTRFL1 interacts with RTBP1 and OsTRBF1 and that these interactions result in the indirect association of OsTRFL1 with telomere repeats in planta.

\section{Abnormal telomere lengthening and developmental defects of ostrfl1}

RT-PCR analysis demonstrated the presence of OsTRFL1 transcripts in most developing tissues examined, including callus, shoots, leaves, panicles, and seeds, except for roots (Fig. 4A). However, the expression of OsTRFL1 was very low in mature stems and seeds, suggesting that OsTRFL1 may play a major role in developing organs in rice. To determine the detailed cellular functions of OsTRFL1, a loss-of-function ostrfl1 mutant line that contained a T-DNA insertion in the fifth exon of the OsTRFL1 gene was isolated (Fig. 4B). A homozygous allele for the T-DNA insertion was identified by genomic PCR with the LP/RP and LB/RP primer sets (Fig. 4C). Genomic Southern blot analysis indicated that the ostrfl1 mutant progeny contained a single T-DNA integration into the OsTRFL1 gene (Fig. 4D). Fig. 4E shows that homozygous ostrfl1 mutant leaves contained markedly reduced, but still detectable, amounts of OsTRFL1 transcript, while the heterozygous ostrfl1 line contained slightly lower transcript levels (Fig. 4E). These results indicated that ostrfl1 is not a null mutant.

To assess whether the reduced level of OsTRFL1 affected telomere maintenance, telomere lengths in wild-type and ostrfl1 plants were measured by the terminal restriction fragments assay (19). The telomere length of wild-type rice was 5-12 kb (Fig. 4F), which is consistent with our previous results (20). In contrast, the telomere lengths of heterozygous and homozygous ostrfl1 mutant plants were 6-25 kb (Fig. 4F). Thus, it appears that OsTRFL1 is involved in the negative regulation of telomere lengthening in rice plants.

To explore the effects of decreased levels of OsTRFL1 and telomere lengthening on the development of rice plants, the phenotypic properties of wild-type and ostrfl1 plants were compared. As presented in Fig. 4G, the homozygous ostrfl1 line exhibited serious growth retardation at the vegetative stage. The mutant plants were also defective in the development of reproductive organs, resulting in sterile flowers without functional seeds. The abnormal phenotypes of the heterozygous ostrfl1 mutant were intermediate between

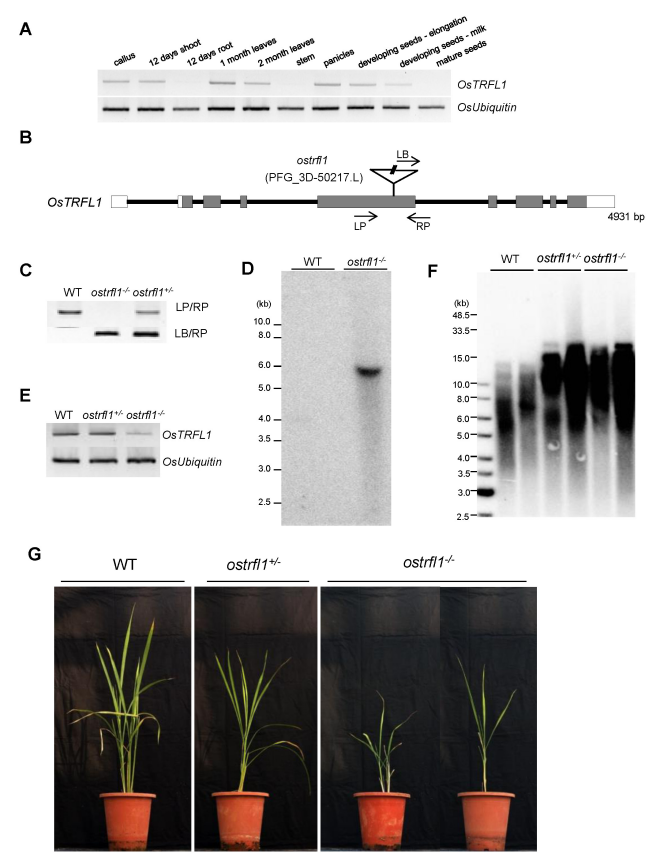

Fig. 4. Phenotypic analysis of a T-DNA-inserted ostrfl1 mutant line. (A) RT-PCR analysis of OsTRFL1. Total RNA was isolated from various rice tissues as indicated and analyzed by RT-PCR. OsUbiquitin was included as a loading control. (B) Schematic structure of the rice ostrfl1 mutant (line PFG_3D-50217.L) with the inserted T-DNA. The inverted triangle indicates the location of the integrated T-DNA in the fifth exon of OsTRFL1 located on chromosome 2. The white bars indicate the 5'- and $3^{\prime}$-untranslated regions. The grey bars represent the coding regions, and the black lines indicate the introns. The T-DNAspecific LB primer and gene-specific primers (LP and RP) used for genotyping PCR are indicated with arrows. (C) Genotyping PCR for ostrfl1. The primer sets used for genomic PCR are shown on the right side of the agarose gel. WT, wild-type; ostrfl1 ${ }^{-1-}$, homozygous allele; ostrfl1 ${ }^{+1-}$, heterozygous allele. (D) Genomic Southern blot analysis. Total leaf genomic DNA was isolated from wild-type (WT) and homozygous ostrfl1 mutant rice plants. The isolated DNA was digested with EcoRV and hybridized to ${ }^{32} \mathrm{P}$-labeled hygromycin B phosphotransferase $(\mathrm{Hph})$ probes under high stringency conditions. (E) Expression levels of OsTRFL1 in wild-type (WT) and homozygous (ostrfl ${ }^{-1-}$ ) and heterozygous (ostrff $^{Y^{\prime-}}$ ) mutant plants. (F) Telomere lengths in wild-type (WT) and ostrfl1 mutant plants. Rice leaf genomic DNA was digested with Taql restriction enzyme, subjected to pulse-field gel electrophoresis, and analyzed by Southern blotting using ${ }^{32}$ P-labeled (TTTAGGG) 70 telomere repeat probes. WT, wild-type; ostrff $1^{-1-}$, homozygote; ostrfl1 $1^{+-}$, heterozygote. (G) The overall morphology of 2-month-old wild-type, heterozygous $\left(\right.$ ostrfl $\left.^{+-1}\right)$, and homozygous (ostrfl ${ }^{-1-}$ ) mutant rice plants grown under greenhouse conditions. 
the wild-type and homozygous mutant plant phenotypes (Fig. $4 G)$.

In conclusion, OsTRFL1, which harbors a single DNA-binding Myb motif in its C-terminus, is associated with plant telomeric repeats in vivo possibly via its interactions with the DS-TBPs RTBP1 and OsTRBF1 in rice. Suppression of OsTRFL1 resulted in abnormal telomere lengthening and developmental anomalies in both vegetative and reproductive growth. Overall, these results indicate that OsTRFL1 plays a critical role in telomere length homeostasis and the normal development of rice plants.

\section{MATERIALS AND METHODS}

\section{Recombinant protein expression and gel retardation assay} The Myc-(His) ${ }_{6}-\mathrm{OsTRFL}^{486-623}$, (His) ${ }_{6}-\mathrm{OsTRBF}^{1-128}$, and Myc(His) ${ }_{6}-\mathrm{RTBP} 1^{506-620}$ recombinant proteins, all of which possess the Myb DNA-binding domain (Supplementary Fig. S2), were expressed in E. coli BL21 (DE3) cells and purified by affinity chromatography using Ni-NTA agarose (Qiagen, Hilden, Germany) according to the manufacturer's protocol. The gel retardation assay was performed as described by Byun and $\operatorname{Kim}(22)$.

\section{Yeast two-hybrid and in vitro pull-down assays}

The yeast two-hybrid assay was performed according to the method of Byun et al. (14), with slight modifications. To investigate the interactions between OsTRFL1 and rice DS-TBPs, full-length OsTRFL1 was ligated into the pGBK T7 vector (Clontech, Mountain View, CA, USA). The full-length coding regions of OsTRBF1, RTBP1, and OsTRFL1 were inserted into the pGAD T7 vector. As negative controls, OsKu70 and T-antigen were also inserted into the pGAD T7 vector. These constructs were co-transformed into AH109 yeast cells. The transformed yeast cells were plated onto three-minus (-Leu/-Trp/-His) medium supplemented with $10 \mathrm{mM}$ 3-amino-1,2,4,-triazole and grown at $30^{\circ} \mathrm{C}$ for 4 days.

Bacterially expressed MBP-OsTRFL1, MBP-OsTRBF1, (His) $6^{-}$ OsTRFL1, (His) ${ }_{6}$-OsTRBF1, and (His) ${ }_{6}-\mathrm{RTBP} 1$ recombinant proteins were purified by affinity chromatography using Ni-NTA agarose (Qiagen) for (His) ${ }_{6}$-tagged proteins and amylose resin (New England Biolabs) for MBP-tagged proteins. In vitro pull-down and immunoblot analyses were performed as described by Byun et al. (26) with anti-His and anti-MBP antibodies (Applied Biological Materials, Richmond, BC, Canada).

\section{In vivo ChIP assay}

The ChIP assay was carried out as described by Byun and Kim (22), with slight modifications. Agrobacterium containing the 35S:OsTRFL1-sGFP, 35S:OsTRBF1-sGFP, 35S:OsRAD51D-sGFP, and 35S:RTBP1-sGFP constructs were infiltrated into tobacco (Nicotiana benthamiana L.) leaves. After $48 \mathrm{~h}$ of infection, the infiltrated leaves were crosslinked with $1 \%$ formaldehyde and then quenched with $125 \mathrm{mM}$ glycine. Chromatin was extracted from the leaves and incubated without (negative control) or with an anti-GFP antibody (1:1000 dilution; Applied Biological Materials). The samples were precipitated with protein G sepharose resin (GE Healthcare, Little Chalfont, United Kingdom), blotted onto a Hybond-N nylon membrane, and hybridized to ${ }^{32}$ P-labeled (TTTAGGG) ${ }_{70}$ telomeric repeats under high stringency conditions.

\section{Subcellular localization and BiFC assays}

The $3^{\prime}$ end of the OSTRFL1 coding region was tagged with synthetic green fluorescent protein (sGFP) in-frame and inserted into the pEarleyGate (pEG) 100 binary vector. The vector was then transformed into Agrobacterium strain LBA4404 by electroporation. The 35S:OsTRFL1-sGFP, 35S:RTBP1-sGFP, 35S:OsTRBF1-sGFP, 35S:NLS-mRFP, and 35S:mRFP-OsTRFL1 constructs were expressed in tobacco leaves using Agrobacteriummediated infiltration. After two days of infection, protoplasts were extracted from the tobacco leaves, and fluorescent protein signals were visualized by fluorescence microscopy (BX51; Olympus, Japan). NLS-mRFP was used as a nuclear marker.

For the BiFC assay, the $3^{\prime}$ end of the OsTRFL1 coding region was tagged with the $155 \mathrm{~N}$-terminal amino acids of YFP $\left(\mathrm{YFP}^{\mathrm{N}}\right)$, and the $3^{\prime}$ ends of OsTRBF1 and RTBP1 were fused to the 86 C-terminal amino acids of YFP $\left(\right.$ YFP $\left.^{\mathrm{C}}\right)$. The fusion constructs were co-expressed in tobacco leaves by Agrobacteriummediated infiltration. Mesophyll cells of the infiltrated leaves were visualized by fluorescence microscopy.

\section{Molecular and phenotypic analysis of the ostrfl1 T-DNA mutant line \\ Identification and analysis of the ostrfl1 mutant were performed according to the method of Byun and Kim (22). For the terminal restriction fragments assay, leaf genomic DNA was isolated from wild-type and ostrfl1 mutant rice plants and then digested with Taql restriction enzyme. The obtained DNA fragments were separated on a $0.7 \%$ agarose gel by pulse-field gel electrophoresis using a CHEFDR III system (Bio-Rad, Hercules, CA, USA) at $6 \mathrm{~V} \mathrm{~m}^{-1}$ for $12 \mathrm{~h}$ at an angle of $120^{\circ}$ with switching times ramped from 1 to $10 \mathrm{~s}$ at $14^{\circ} \mathrm{C}$. The DNA gel blot was hybridized to a ${ }^{32}$ P-labeled (TTTAGGG) 70 telomere repeat probe. Hybridization signals were visualized by autoradiography at $-80^{\circ} \mathrm{C}$.}

\section{ACKNOWLEDGEMENTS}

This work was supported by a grant from the Woo Jang Chun Special Project (grant number PJ009106 to W.T.K.) funded by the Rural Development Administration, Republic of Korea. M.Y.B. was supported by a post-doctoral research grant from the Basic Science Research Program through the National Research Foundation of Korea (NRF) funded by the Ministry of Education (grant number NRF-2014R1A6A3A01056186). 


\section{CONFLICTS OF INTEREST}

The authors have no conflicting interests.

\section{REFERENCES}

1. O'Sullivan RJ and Karlseder J (2010) Telomeres: protecting chromosomes against genome instability. Nat Rev Mol Cell Biol 11, 171-181

2. Doksani Y and de Lange T (2014) The role of doublestrand break repair pathways at functional and dysfunctional telomeres. Cold Spring Harb Perspect Biol 6, a016576

3. Procházková Schrumpfová $P$, Schořová Š and Fajkus J (2016) Telomere- and telomerase-associated proteins and their functions in the plant cell. Front Plant Sci 7, 851

4. Nandakumar J and Cech TR (2013) Finding the end: recruitment of telomerase to telomeres. Nat Rev Mol Cell Biol 14, 69-82

5. Fouquerel E, Parikh D and Opresko P (2016) DNA damage processing at telomeres: The ends justify the means. DNA Repair 44, 159-168

6. Martínez P and Blasco MA (2015) Replicating through telomeres: a means to an end. Trends Biochem Sci 40, 504-515

7. Sfeir A and de Lange T (2012) Removal of shelterin reveals the telomere end-protection problem. Science 336, 593-597

8. Bandaria JN, Qin P, Berk V, Chu S and Yildiz A (2016) Shelterin protects chromosome ends by compacting telomeric chromatin. Cell 164, 735-746

9. Zellinger B and Riha K (2007) Composition of plant telomeres. Biochim Biophys Acta 1769, 399-409

10. Du H, Wang YB, Xie Y et al (2013) Genome-wide identification and evolutionary and expression analyses of MYB-related genes in land plants. DNA Res 20, 437-448

11. Mozgová I, Schrumpfová PP, Hofr C and Fajkus J (2008) Functional characterization of domains in AtTRB1, a putative telomere-binding protein in Arabidopsis thaliana. Phytochemistry 69, 1814-1819

12. Dvorácková $M$, Rossignol $\mathrm{P}$, Shaw PJ, Koroleva OA, Doonan JH and Fajkus J (2010) AtTRB1, a telomeric DNA-binding protein from Arabidopsis, is concentrated in the nucleolus and shows highly dynamic association with chromatin. Plant J 61, 637-649

13. Marian CO, Bordoli SJ, Goltz M et al (2003) The maize single myb histone 1 gene, Smh1, belongs to a novel gene family and encodes a protein that binds telomere DNA repeats in vitro. Plant Physiol 133, 1336-1350
14. Byun MY, Hong JP and Kim WT (2008) Identification and characterization of three telomere repeat-binding factors in rice. Biochem Biophys Res Commun 372, 85-90

15. Schrumpfová PP, Vychodilová I, Dvořáčková $M$ et al (2014) Telomere repeat binding proteins are functional components of Arabidopsis telomeres and interact with telomerase. Plant J 77, 770-781

16. Lee WK and Cho MH (2016) Telomere-binding protein regulates the chromosome ends through the interaction with histone deacetylases in Arabidopsis thaliana. Nucleic Acids Res 44, 4610-4624

17. Ko S, Jun $\mathrm{SH}$, Bae $\mathrm{H}$ et al (2008) Structure of the DNA-binding domain of NgTRF1 reveals unique features of plant telomere-binding proteins. Nucleic Acids Res 36, 2739-2755

18. Ko S, Yu EY, Shin J et al (2009) Solution structure of the DNA binding domain of rice telomere binding protein RTBP1. Biochemistry 48, 827-838

19. Yang SW, Kim SK and Kim WT (2004) Perturbation of NgTRF1 expression induces apoptosis-like cell death in tobacco BY-2 cells and implicates NgTRF1 in the control of telomere length and stability. Plant Cell 16, 3370-3385

20. Hong JP, Byun MY, Koo DH et al (2007) Suppression of rice telomere binding protein 1 results in severe and gradual developmental defects accompanied by genome instability in rice. Plant Cell 19, 1770-1781

21. Karamysheva ZN, Surovtseva YV, Vespa L, Shakirov EV and Shippen DE (2004) A C-terminal Myb extension domain defines a novel family of double-strand telomeric DNA-binding proteins in Arabidopsis. (2004) J Biol Chem 279, 47799-47807

22. Byun MY and Kim WT (2014) Suppression of OsRAD51D results in defects in reproductive development in rice (Oryza sativa L.). Plant J 79, 256-269

23. Renfrew KB, Song $X$, Lee JR, Arora A and Shippen DE (2014) POT1a and components of CST engage telomerase and regulate its activity in Arabidopsis. PLoS Genet 10, e1004738

24. Miyagawa K, Low RS, Santosa V et al (2014) SUMOylation regulates telomere length by targeting the shelterin subunit Tpz1 (Tpp1) to modulate shelterin-Stn1 interaction in fission yeast. Proc Natl Acad Sci U S A 111, 5950-5955

25. Kim MK and Kim WT (2018) Telomere structure, function, and maintenance in plants. J Plant Biol 61, 131-136

26. Byun MY, Cui LH and Kim WT (2015) Suppression of OsKu80 results in defects in developmental growth and increased telomere length in rice (Oryza sativa L.). Biochem Biophys Res Commun 468, 857-862 